\title{
APLICACIÓN DE LA TÉCNICA SMED EN EL PROCEDIMIENTO DE CAMBIO DE TINTAS DE LA REFERENCIA BOLSA KRAFF COLANTA ENTERA 3C A BOLSA KRAFF AMTEX TANNUS 2C
}

\author{
ApPliCATION OF THE SMED TECHNIQUE IN THE PROCESS OF \\ CHANGE OF INKS FROM THE KRAFF WHOLE COLANTA 3C BAG \\ REFERENCE TO KRAFF AMTEX TANNUS 2C BAG
}

${ }^{1}$ Fernando Ulpiano Pantoja Agreda, ${ }^{2}$ Jorge Humberto Castrillón,

\begin{abstract}
Universidad Nacional Abierta y a Distancia, Escuela de las ciencias básicas tecnología e ingeniería, Medellín, Colombia,
${ }^{1}$ fepantoja@unad.edu.co

${ }^{2}$ jorgeb0913@hotmail.com, orcid.org/0000-0002-3449-9626
\end{abstract}

Recibido: 27/09/2016 • Aprobado: 30/10/2016

\begin{abstract}
RESUMEN
Uno de los procesos significativos de la empresa Occidental de Empaques S.A. es la fabricación de empaques de papel multipliego para la industria cementera, química y alimenticia. El proceso de impresión del empaque requiere del cambio de tintas que redundan en pérdidas de tiempo debido al alistamiento de equipos para el cambio de referencias. Acorde a las consultas establecidas; Single-Minute Exchange of Die (Smed) es una técnica o metodología que busca reducir los tiempos de cambios y lograr realizar cada actividad en menos de 10 minutos. En este trabajo se aplicó la técnica Smed para buscar una oportunidad de mejora y estandarizar el proceso de impresión, lo que se traduce en beneficios de reducción de tiempos e incremento de la productividad. Con la aplicación de esta técnica se minimizaron los tiempos de cuadre un 20\%, esto equivale al 25\% del tiempo programado de máquina mensual, logrando con esta reducción de tiempo un incremento en la productividad del 10\% convertido en unidades de empaques fabricados en 200.000 unidades. Esto genera a la empresa mayor eficiencia y productividad en el proceso logrando una optimización del recurso máquina y mano de obra.
\end{abstract}

Palabras clave: impresión papel multipliego, intercambio del dado, justo a tiempo, mantenimiento productivo total, sistema de producción total.

\section{Abstract}

One of the relevant processes of the Packaging Western Company S.A. is the manufacture of multi-sheet paper packaging for cement, chemical and food industry. The printing process of the packaging requires the change of inks that results in loss of time due to the enlistment of equipment for the change of references. According to 
established consultations, Single-Minute Exchange of Die (SMED) is a technique or methodology that seeks to reduce change times and achieve each activity in less than 10 minutes. In this work the SMED technique was applied to look for an opportunity to improve and standardize the printing process, which translates into benefits of time reduction and productivity increase. With the application of this technique, square times were minimized $20 \%$, this is equivalent to $25 \%$ of the programmed monthly time of the machine, achieving with this reduction of time, an increase in productivity of 10\%, converted into200,000 units of manufactured packaging. This generates to the company greater efficiency and productivity in the process, achieving an optimization of the machine resource and labor.

Keywords: die exchange, Just in time, multi-sheet paper printing, total production system, total productive maintenance.

\section{INTRODUCCIÓN}

El proceso de impresión de empaques de papel es realizado en la tubuladora NL, la cual es una máquina diseñada para la fabricación de tubos de papel multipliegos, los que luego pasan a un segundo proceso para realizar los fondos y finalmente obtener un empaque de papel. La tubuladora NL está asignada en la línea \# 2 como máquina \# 1 donde inicia el proceso de conversión del papel, el cual pasa por la impresora flexográfica y reproduce un logo o imagen en un sustrato (papel o cartón), con tintas a base de agua de rápido secado; luego pasa con unos rodillos de enhebre y se distribuyen las capas de papel para aplicarles el engome o adhesivo, los cortes y por medio de presión se separan las unidades según la referencia, luego pasa por la banda de salida donde está sometida a presión y finalmente se forma un paquete de 20 a 50 unidades para ser arrumado en una estiba para pasar al segundo proceso.

Para nadie es un secreto que las empresas de producción deben estar atentas a una cantidad de desarrollos y necesidades del medio, estas condiciones obligan a los trabajadores a proponer alternativas diferentes, referente a nuevos productos, diseños y/o actualizaciones de los existentes, mediante algunas mejoras puntuales; es así como se sostienen las empresas en entornos competitivos. Parafraseando a Sendoya Diego hace algunas décadas, el único objetivo realmente importante en el control de procesos era lograr alcanzar una operación estable de una parte de la planta, proceso o equipo; sin embargo, hoy en día, la existencia de un mercado, tan variable y difícil de predecir, ha obligado a las empresas a mejorar sus procesos productivos con el fin de mantenerse competitivas y rentables (Sendoya 2013). En las grandes industrias se fomentan prácticas Lean; que corresponden a estructuras de clase mundial, y en ellas el Justo a Tiempo (JIT), Mantenimiento Productivo Total (TPM), Manufactura de clase Mundial (WCM), normas ISO, entre otras, exigen de herramientas probadas y comprobadas en el entorno internacional como se puede observar en el trabajo de B. Ultas (2011), que deben ser replicadas en todos los procesos tanto por sus dependencias como por sus contratistas, lo que obliga a las cadenas productivas a interiorizar los conceptos y aplicarlos para un mayor beneficio tanto de los procesos como de los productos; que a su vez se reflejen en la eficacia y eficiencia de las empresas.

Una de las herramientas de clase mundial que facilita disminuir los tiempos de cambio de línea de producción o de utensilios propios de una referencia o producto es, según Azizi \& Manoharan (2015) Single Minute Exchange of Die Smed; por sus siglas en inglés, y que traduce cambio de troqueles en menos de diez minutos; en este mismo artículo el autor se refiere a Smed como una herramienta que está ligada a reducir los Stocks y mejorar los tiempos de entrega; al reducir los tiempos de cambio, mejora la capacidad de realizar más cambios de modelo o referencia, lotes más ajustados, 
planificar entregas y disminuir el almacenamiento de productos terminados; además se puede pensar como menor consumo de recursos, ligado al tiempo de disponibilidad hombre-máquina.

Autores como M. A. Almomani, M. Aladeemy, A. Abdelhadi \& A. Mumani (2013) consideran que Smed puede mejorar su definición inicial y disminuir esos tiempos de menos de un digito, mediante el apoyo en otras técnicas adicionadas a la metodología propuesta por el ingeniero Shigeo Shingo, su propuesta incluye un enfoque sistémico, que incorpora técnicas de toma de decisión y otros factores presentes como; costos, consumo energético, seguridad, ciclo de vida, mantenimiento entre otros; y es orientado a la flexibilización de la producción.

Observando el trabajo desarrollado por los ingenieros Sugimori, Kusunoki \& Oho (1997) es como se puede interpretar los conceptos de manufactura de clase mundial y más concretamente Total Production Sistem (TPS) en dónde Smed logra un espacio merecido.

Una aplicación dirigida a la industria flexográfica, que permite evaluar factores que intervienen en la calidad y eficiencia del proceso utilizando Smed, es garantía de que esta metodología es adecuada para ser tenida presente en nuestro medio (Lipiak 2017).

Este proyecto se desarrollará siguiendo las pautas establecidas en la técnica Smed, que tiene por objetivo la reducción del tiempo de cambio entre la última pieza producida del producto "A" y la primera pieza producida del producto "B", en una misma línea de producción, que cumple con las especificaciones dadas. El logro de un menor tiempo de cambio, prepara a los operarios afrontar retos similares en otros campos de la planta, lo cual constituye una importante ventaja de carácter secundario de Smed y que se describe por M. Suárez \& M. Dávila (2008).

El punto ideal en este estudio, se concreta en el cambio de la referencia bolsa Kraff Colanta Entera3C a bolsa Kraff Amtex Tannus 2C, lo cual se logra recopilando información de cada cambio realizado en el año 2015.
Se toman cinco muestras y se analizan detalladamente las tareas y los tiempos para cada una de ellas, luego de levantar toda esta información se pasó a aplicar Smed, realizando una separación de las actividades y perfeccionando las operaciones internas y operaciones externas para así minimizar en número de actividades durante el tiempo de paro de la máquina, finalmente se estandarizan esas actividades y se realiza un seguimiento, tomando y registrando tiempos que se comparan con los anteriores para valorar lo propuesto.

Para la reproducción de impresiones se requieren dos materiales, el papel natural que según K. Marsh \& B. Bugusu (2007) es el más resistente y también es utilizado comúnmente para bolsas y envolturas de alimentos; las principales características del papel kraff utilizado para bolsas a fabricar son:

Peso base 85 g $7 \mathrm{~m} 2$, fibra larga y virgen, semi extensible, buen desempeño para impresión.

La otra condición requerida, son las tintas que según M. Rentzhog \& A. Fogden (2006), Cuando se formula la tinta a base de agua, es importante elegir la mezcla correcta de polímeros para obtener el equilibrio requerido de propiedades. Una buena solubilidad es decisiva para obtener imágenes limpias y nítidas en tiradas largas.

Las principales características de la tinta a base agua para impresión flexográfica son la resistencia mecánica; además de un comportamiento adecuado del color, intensidad, tonalidad, brillo, poder de cobertura, limpieza de tono, rápido secado.

\section{Materiales y Métodos}

La necesidad de lograr un menor tiempo se deduce de que, reduciendo los tiempos de preparación se podría minimizar el tamańo de los lotes y por consiguiente reducir los stocks para trabajar en series cortas de productos; situación contemplada en el ejemplo de las plantas de producción de automóviles, donde la fabricación de un coche corresponde al pedido que un 
cliente ha efectuado en algún lugar del mundo; así, un automóvil puede ser de color granate, mientras que la unidad siguiente puede ser de color verde.

La filosofía Smed, vista como eliminar el concepto de lote de fabricación reduciendo al máximo el tiempo de preparación de máquinas y de materiales, garantiza el pilar de mejoras enfocadas de TPM y nos permite acorde con N. H. Kamath \& L. L. R. Rodrigues (2006), aproximarnos a lo que se considera un modelo de impresión eficiente, según la dinámica de sistemas.

Pensando en Smed como herramienta de mejora continua, enfocada en la gente de cada proceso y en la estandarización del proceso, como lo plantean M. Suárez \& M. Dávila (2008), "la movilización y la participación de los empleados, genera un canal o un medio para que los mismos, puedan contribuir al desarrollo de la compañía”; con el objetivo principal de incrementar la productividad mediante la reducción de tiempos de las operaciones en los diferentes ciclos del proceso.

En nuestro caso los cambios de color de tintas para cada referencia de empaques, ocupan un $20 \%$ del tiempo programado de máquina y se pueden realizar al mes 35 cambios, con un significativo tiempo de paro; generando como consecuencia baja productividad. Con la implementación de Smed en este punto específico del proceso, se busca reducir el índice de tiempo de paro de máquina en la actividad de cambios de tintas y así generar a la empresa mayor eficiencia y productividad en el proceso, logrando optimizar el recurso máquina y mano de obra con el impacto en los demás indicadores que emerjan de su implementación.

\section{A. Metodología.}

Acorde a la definición de Smed que realizan A. Abraham, K. N. Ganapathi, \& K. Motwani (2012) se debe realizar los siguientes pasos:

1. Etapa preliminar: estudio de la operación de cambio.

2. Primera etapa: separar tareas internas y externas.

3. Segunda etapa: convertir las tareas internas en externas.

4. Tercera etapa: perfeccionar las tareas internas y externas.

\section{1) Etapa preliminar: estudio de la operación de cambio}

Descripción del procedimiento para el cambio de tintas utilizadas en la referencia bolsa kraff colanta entera 3C a bolsa kraff amtex tannus 2C. Después de realizar los análisis y mediciones de tiempo por operación del procedimiento para el cambio de tintas de la referencia bolsa Kraff Colanta Entera 3C a bolsa Kraff Amtex Tannus 2C y tomando información de cinco de los últimos cambios del 2015, recopilando datos desde los informes de producción y evidenciados en registros de filmación de circuito cerrado de televisión, se obtienen los siguientes datos a mencionar, de la máquina con impresora flexográfica de cuatro colores para tinta a base agua, con alimentación de tinta por medio de bombas neumáticas, recepción de tintas en bandejas de acero inoxidable y dosificación de tintas por medio de rodillos de caucho y rodillo entintador cerámico.

La fotografía 1, muestra la estructura física de la impresora New Long flexográfica de cuatro estaciones de impresión, encargada de reproducir las imágenes y textos en el empaque.

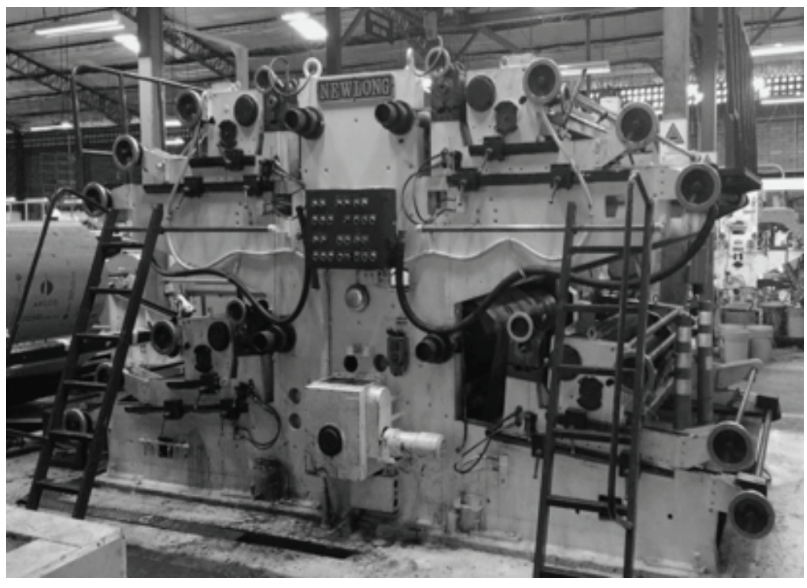

Fotografía. 1 Impresora New Long flexográfica de cuatro estaciones de impresión.

Fuente: autores

La fotografía 2, enseña la estación de impresión \#1 con cada una de sus partes: bandeja, rodillo entintador, rodillo cerámico, y tambor porta clise (foto polímero). 


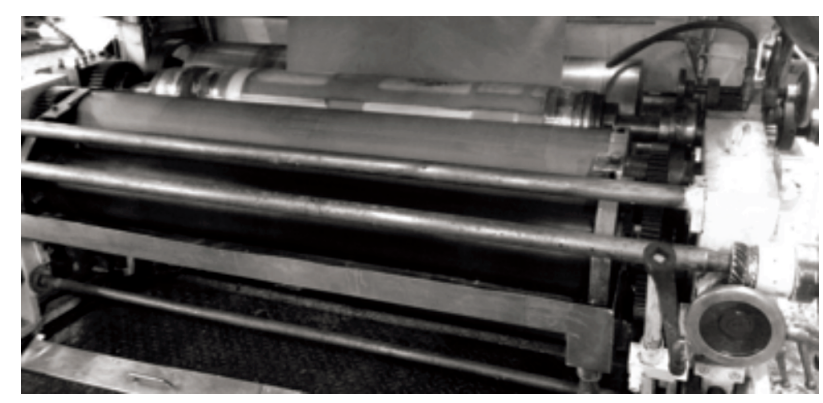

Fotografía. 2 Estación de impresión \#1

Fuente: autores

La fotografía 3, ilustra el sistema de alimentación de tintas con bombas neumáticas.

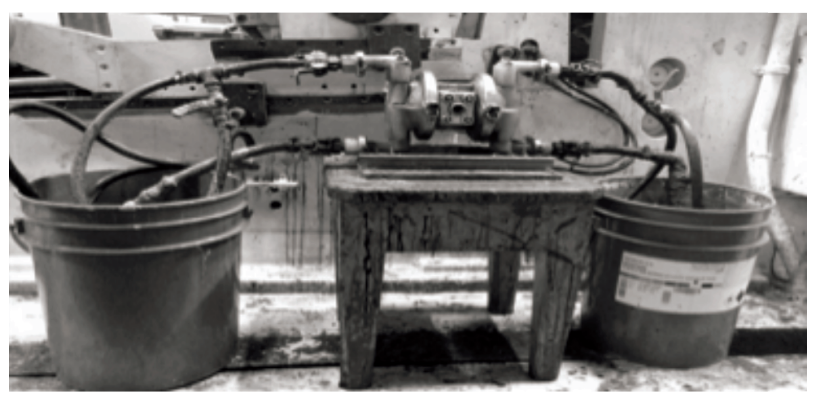

Fotografía. 3 Sistema de alimentación de tintas y bombas neumáticas

Fuente: autores

Descripción del proceso cambio de tintas utilizadas en la referencia bolsa kraff Colanta entera 3c a bolsa kraff amtex tannus 2c. El proceso de fabricación de bolsa de papel multipliego para la industria cementara, química y alimenticia, se realiza en una tubuladora New Long con impresora flexográfica de cuatro colores. En el proceso de impresión se realiza el cambio de color de tinta, cada que se cambia de referencia y/o de cliente, en este caso son las referencias bolsa kraff Colanta entera 3c a bolsa kraff amtex tannus 2c. Por lo cual es necesario cambiar las siguientes referencias de tintas y realizar las siguientes actividades propuestas en la tabla 1 y en la figura 1 .

En esta tabla 1 se mencionan las referencias de tintas que se aplican en el empaque asociado bolsa kraff Colanta entera 3c y bolsa kraff amtex tannus 2c.

TABla 1

Referencias de tintas que se aplican en la bolsa kraff Colanta entera $3 \mathrm{c}$ y bolsa kraff amtex tannus $2 \mathrm{c}$

\begin{tabular}{|c|c|c|}
\hline \multicolumn{3}{|c|}{ BOLSA KRAFF COLANTA ENTERA 3C } \\
\hline $\begin{array}{l}\text { Estación de } \\
\text { Impresión }\end{array}$ & Referencia tinta & Proveedor \\
\hline 1 & 20659: WB Verde 1:FK24 & Tintas S. A \\
\hline 2 & $\begin{array}{l}\text { 80224: WB Naranja GCMI } \\
\text { 80ED: FK24 }\end{array}$ & Tintas S. A \\
\hline 3 & Negro 90INTEC & Grupo Sánchez \\
\hline \multicolumn{3}{|c|}{ BOLSA KRAFF AMTEX TANNUS 2C } \\
\hline $\begin{array}{l}\text { Estación de } \\
\text { Impresión }\end{array}$ & Referencia tinta & Proveedor \\
\hline 1 & 704B007: WB Azul: FK24 & Tintas S. A \\
\hline 2 & $\begin{array}{l}\text { 80110: WB Amarillo 10-1: } \\
\text { FK24 }\end{array}$ & Tintas S. A \\
\hline 3 & 70306: WB Rojo: FK24 & Tintas S. A \\
\hline
\end{tabular}

Fuente: elaboración propia

La fig. 1, describe, por medio de un flujograma de procesos, cada una de las tareas desarrolladas en el cambio de tintas que se aplican en la referencia de empaques bolsa kraff Colanta entera 3c y bolsa kraff amtex tannus $2 c$. 


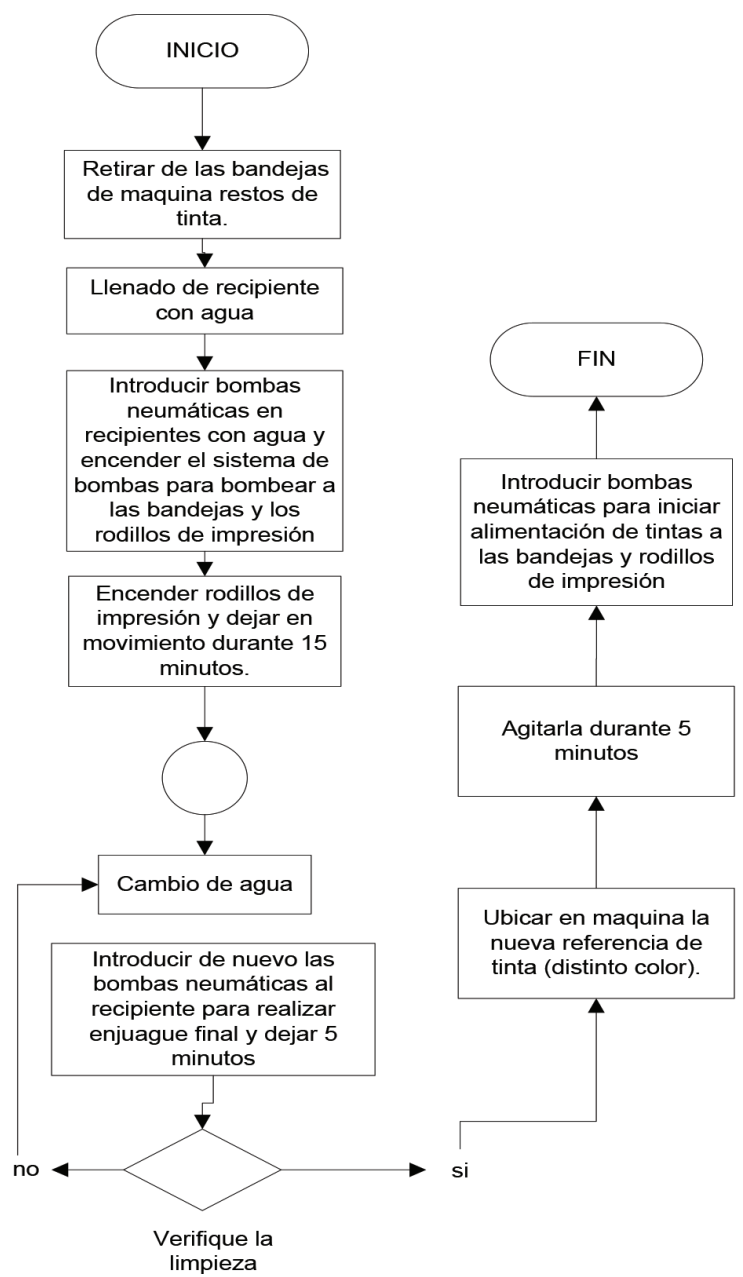

Fig. 1 Flujograma de procesos para el cambio de tintas.

Fuente: los autores

En la fig. 2, se muestra el comportamiento promedio de los cinco cambios de tinta tomados como muestra en el 2015, con tiempo analizados en minutos.

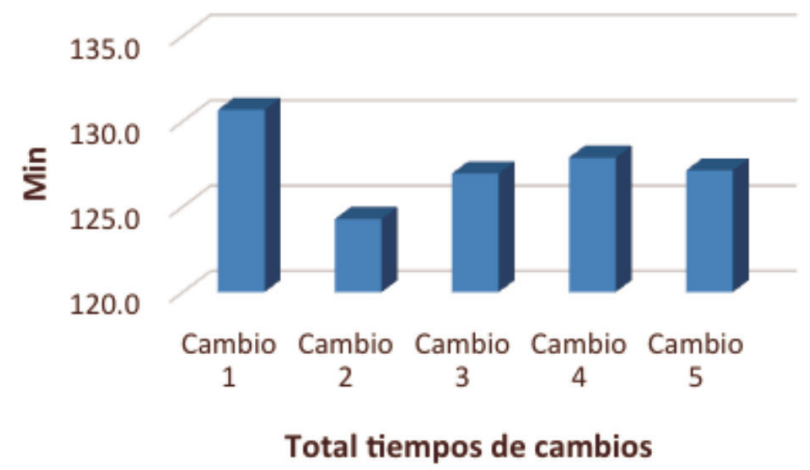

Fig. 2 Comportamiento promedio de los cinco cambios de tinta. Fuente: elaboración propia
En la fig. 3, se muestra la tendencia de las actividades que ocupan el mayor tiempo en el cambio 1-2015 como se ve reflejado en las columnas 4-6-8. Unidad de medida minutos.

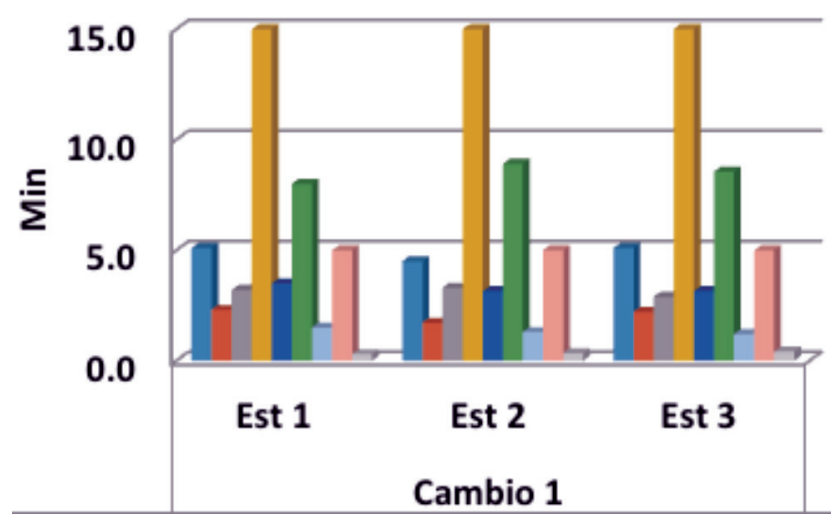

Fig. 3 Tendencia de las actividades que ocupan el mayor tiempo en el cambio 1-2015

Fuente: elaboración propia

En la fig. 4, se muestra la tendencia de las actividades que ocupan el mayor tiempo en el cambio 2-2015 como se ve reflejado en las columnas 4-6-8. Unidad de medida minutos.

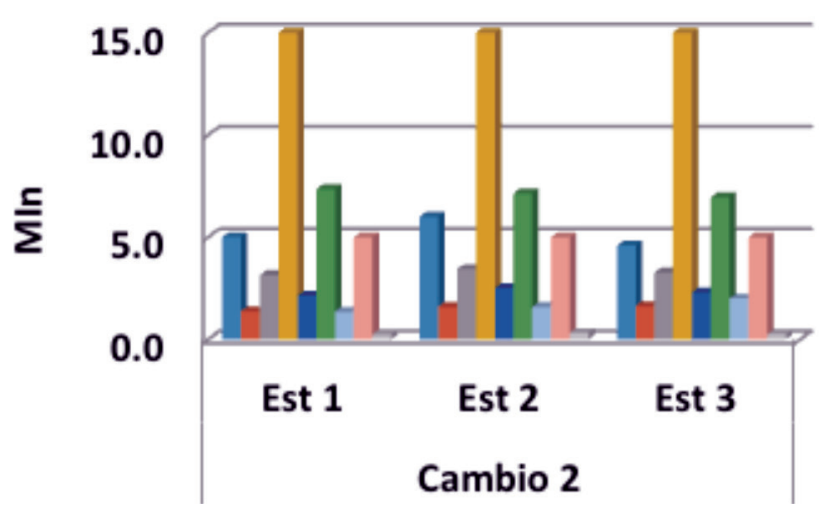

Fig. 4 Tendencias de las actividades que ocupan el mayor tiempo en el cambio 2-2015

Fuente: elaboración propia

En esta fig. 5, se muestra la tendencia de las actividades que ocupan el mayor tiempo en el cambio 3-2015 como se ve reflejado en las columnas 4-6-8. Unidad de medida minutos. 


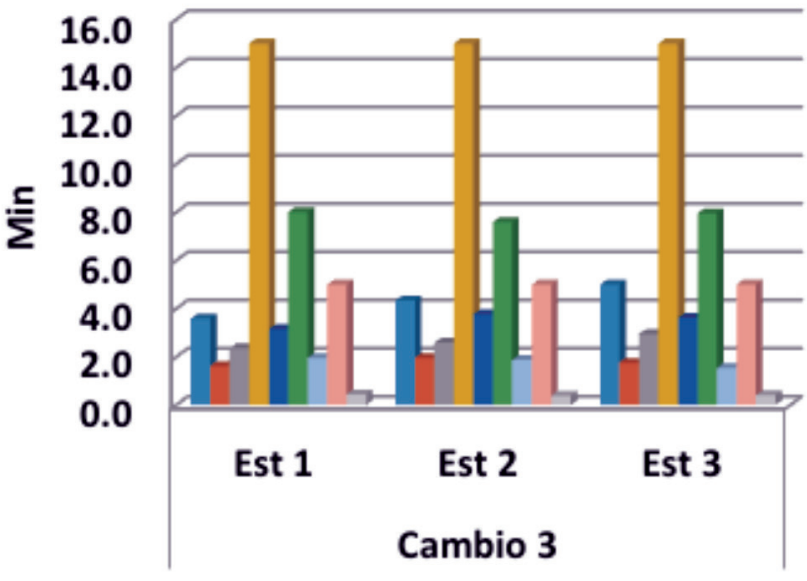

Fig. 5 Tendencias de las actividades que ocupan el mayor tiempo en el cambio 3-2015

La fig. 6, muestra la tendencia de las actividades que ocupan el mayor tiempo en el cambio 4-2015 como se ve reflejado en las columnas 4-6-8.

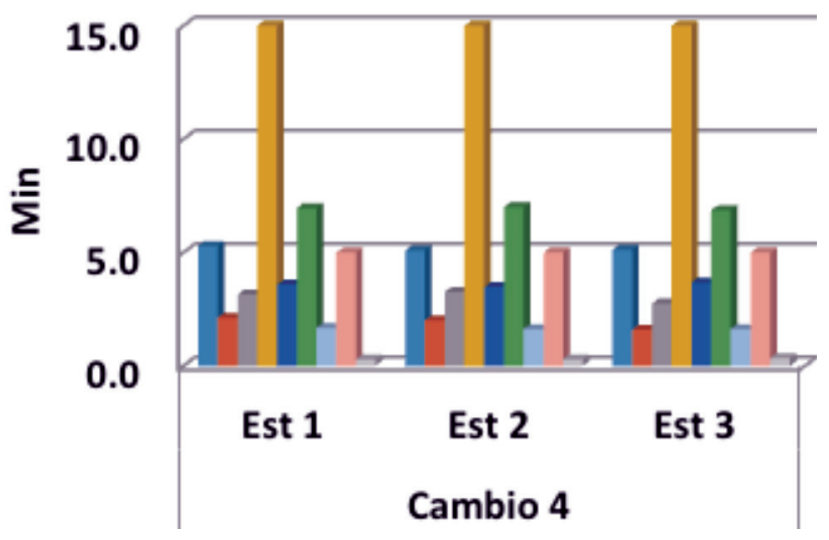

Fig. 6 Tendencias de las actividades que ocupan el mayor tiempo en el cambio 4-2015

Fuente: elaboración propia

La fig. 7, muestra la tendencia de las actividades que ocupan el mayor tiempo en el cambio 5-2015 como se ve reflejado en las columnas 4-6-8. Unidad de medida minutos.

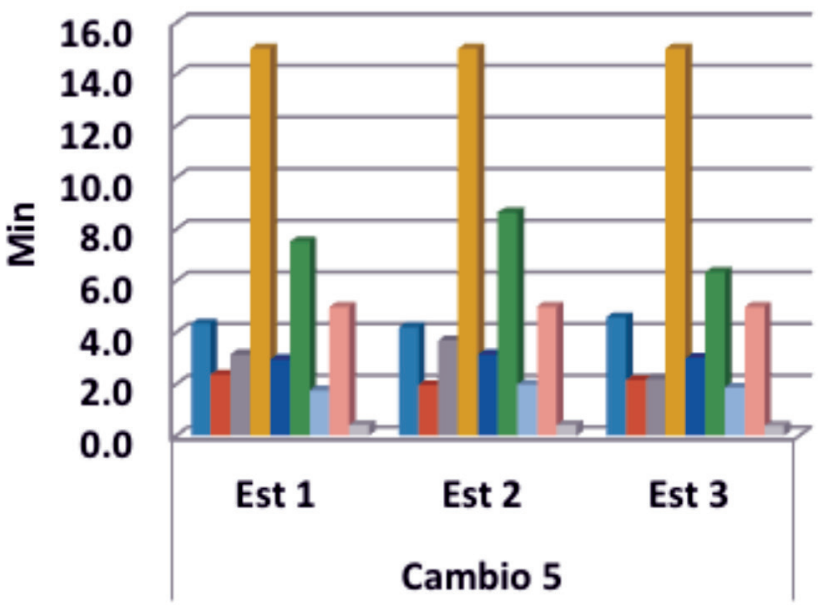

Fig. 7 Tendencias de las actividades que ocupan el mayor tiempo en el cambio 5-2015

Fuente: elaboración propia

\section{2) Primera etapa: separar tareas internas y externas.}

Descripción del procedimiento para el cambio de tintas de la referencia bolsa Kraff Colanta Entera 3C a bolsa Kraff Amtex Tannus 2C aplicando la técnica Smed.

Ahora se aplicará la técnica Smed en el procedimiento para el cambio de tintas de la referencia bolsa Kraff Colanta Entera 3C a bolsa Kraff Amtex Tannus 2C, cambiando algunas actividades y luego definiendo y convirtiendo algunas de las actividades internas en externas para lograr la reducción de tiempos de paro de máquina.

En esta tabla 2 se mencionan cada una de las referencias de tintas que se aplican en el empaque bolsa kraff Colanta entera 3c y bolsa kraff amtex tannus 2c. 
TABLA 2

Tintas aplicadas a bolsa kraff Colanta entera 3c y bolsa kraff amtex tannus 2c.

\begin{tabular}{|c|c|c|}
\hline \multicolumn{3}{|c|}{ BOLSA KRAFF COLANTA ENTERA 3C } \\
\hline $\begin{array}{l}\text { Estación de } \\
\text { Impresión }\end{array}$ & Referencia tinta & Proveedor \\
\hline 1 & 20659: WB Verde 1:FK24 & Tintas S. A \\
\hline 2 & $\begin{array}{l}\text { 80224: WB Naranja } \\
\text { GCMI 80ED: FK24 }\end{array}$ & Tintas S. A \\
\hline 3 & Negro 90INTEC & Grupo Sánchez \\
\hline \multicolumn{3}{|c|}{ BOLSA KRAFF AMTEX TANNUS 2C } \\
\hline $\begin{array}{l}\text { Estación de } \\
\text { Impresión }\end{array}$ & Referencia tinta & Proveedor \\
\hline 1 & 704B007: WB Azul: FK24 & Tintas S. A \\
\hline 2 & $\begin{array}{l}\text { 80110: WB Amarillo 10-1: } \\
\text { FK24 }\end{array}$ & Tintas S. A \\
\hline 3 & 70306: WB Rojo: FK24 & Tintas S. A \\
\hline
\end{tabular}

Fuente: elaboración propia

En la tabla 3, se describen cada una de las operaciones del cambio de tinta para el cambio de referencia bolsa kraff Colanta entera $3 \mathrm{c}$ a bolsa kraff amtex tannus $2 \mathrm{c}$. Aplicando la técnica Smed, separando las actividades en internas y externas.

\section{TABLA 3}

Operaciones de cambio de tinta entre referencias. Aplicando la técnica Smed

\begin{tabular}{|c|c|c|c|}
\hline & ACTIVIDAD & INTERNA & EXTERNA \\
\hline 1 & $\begin{array}{l}\text { Retirar de las bandejas de } \\
\text { máquina restos de tinta }\end{array}$ & $\mathrm{X}$ & \\
\hline 2 & $\begin{array}{l}\text { Llenado de bomba } \\
\text { atomizadora }\end{array}$ & & $\mathrm{X}$ \\
\hline 3 & $\begin{array}{l}\text { Aplicar } 10 \text { litros de agua en } \\
\text { a la superficie de los rodillos } \\
\text { y bandeja con la bomba } \\
\text { atomizadora }\end{array}$ & $\mathrm{X}$ & \\
\hline 4 & $\begin{array}{l}\text { Secar rodillos y bandeja } \\
\text { con paño (Wypall) }\end{array}$ & $\mathrm{X}$ & \\
\hline 5 & $\begin{array}{l}\text { Llenar recipiente con } 10 \\
\text { litros de agua }\end{array}$ & & $\mathrm{X}$ \\
\hline 6 & $\begin{array}{l}\text { Lavado sistema alimentación } \\
\text { tintas introducir bomba } \\
\text { neumática en el recipiente } \\
\text { encender y bombear } 3 \mathrm{~min}\end{array}$ & $\mathrm{X}$ & \\
\hline 7 & Botar agua sucia & $\mathrm{X}$ & \\
\hline 8 & $\begin{array}{l}\text { Ubicar en máquina la } \\
\text { nueva referencia de tinta } \\
\text { (distinto color). }\end{array}$ & & $\mathrm{X}$ \\
\hline 9 & Agitarla durante 5 minutos. & & $\mathrm{X}$ \\
\hline 10 & $\begin{array}{l}\text { Introducir bombas neumáti- } \\
\text { cas para iniciar alimentación } \\
\text { de tintas a las bandejas y } \\
\text { rodillos de impresión }\end{array}$ & $\mathrm{X}$ & \\
\hline
\end{tabular}

Fuente: elaboración propia 


\section{3) Segunda etapa: convertir las tareas internas y externas.}

A continuación podremos observar las gráficas ilustrativas del cambio de referencia de tintas con la aplicación Smed. Aquí se tienen solo las columnas que nos muestran los tiempos de las actividades internas del cambio de las tintas, en el tiempo que la maquina estará parada en dicha actividad.

En la Fig. 8, la gráfica muestra la tendencia de las actividades que ocupan el mayor tiempo en el cambio de tintas.1-2016 como se ve reflejado en las columnas 1-3-6. Unidad de medida minutos.

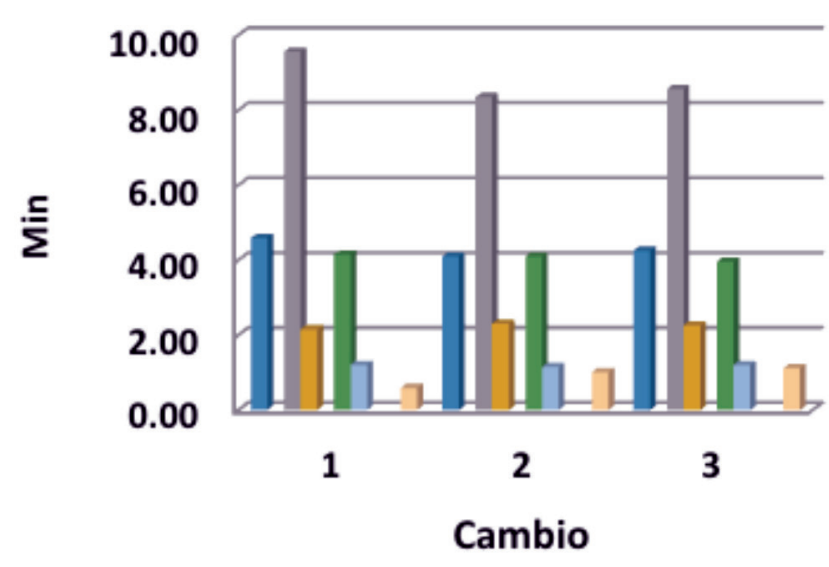

Fig. 8 Tendencia de las actividades que ocupan el mayor tiempo en el cambio de tintas. 1-2016

Fuente: elaboración propia

En la Fig. 9, se muetra la tendencia de las actividades que ocupan el mayor tiempo en el cambio de tintas 2-2016 como se ve reflejado en las columnas 1-3-6. Unidad de medida minutos.

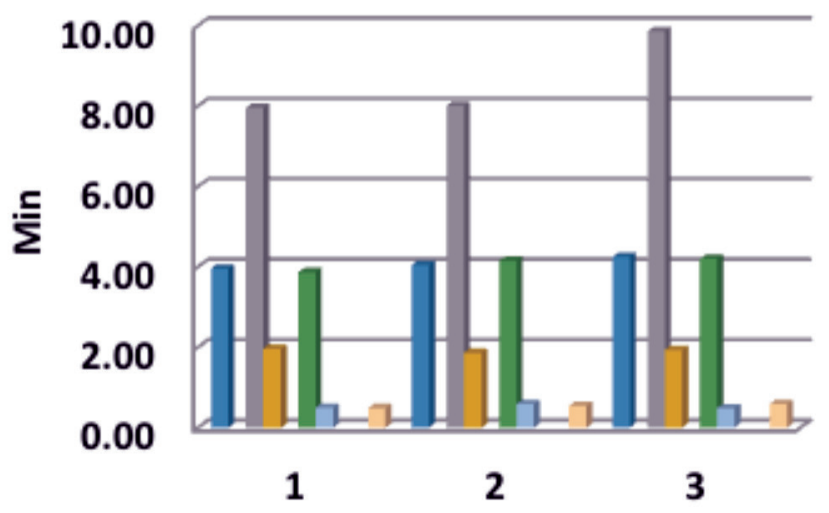

Fig.9 Tendencia de las actividades que ocupan el mayor tiempo en el cambio de tintas. 2-2016

Fuente: elaboración propia

En esta la Fig. 10 se muestra la tendencia de las actividades que ocupan el mayor tiempo en el cambio tintas 3-2016 como se ve reflejado en las columnas 1-3-6. Unidad de medida minutos.

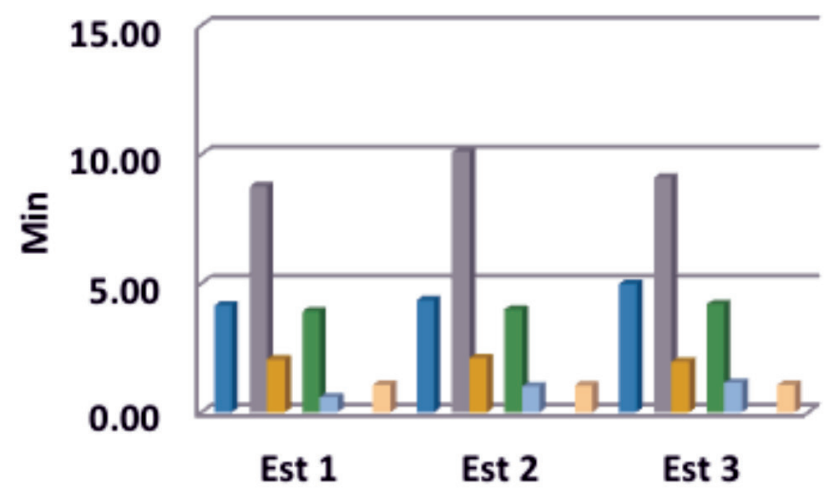

Fig.10 Tendencia de las actividades que ocupan el mayor tiempo en el cambio tintas 3-2016.

Fuente: elaboración propia 
La Fig. 11 muestra la tendencia de las actividades que ocupan el mayor tiempo en el cambiode tintas 4-2016 como se ve reflejado en las columnas 1-3-6. Unidad de medida minutos.

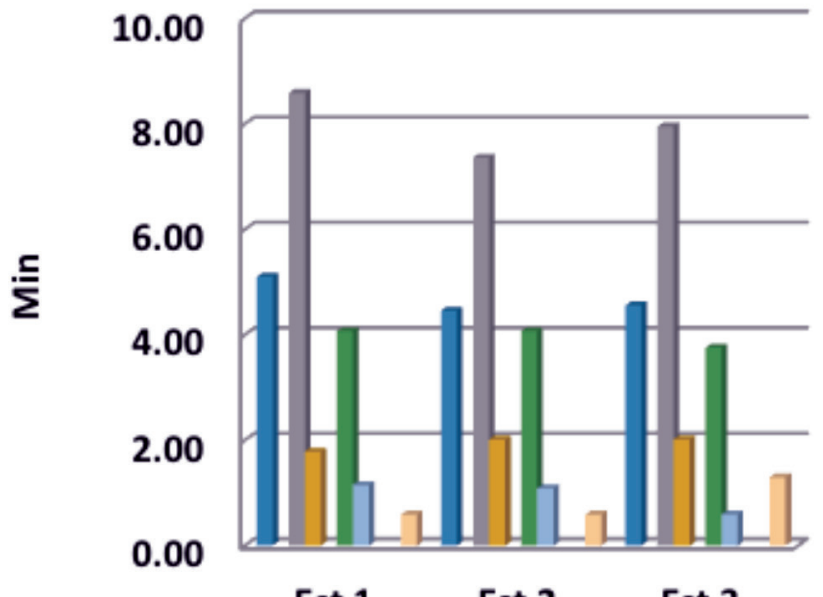

Fig.11 Tendencia de las actividades que ocupan el mayor tiempo en el cambio tintas 4-2016.

Fuente: elaboración propia

En la Fig. 12 se muestra la tendencia de las actividades que ocupan el mayor tiempo en el cambio de tintas 5-2016 como se ve reflejado en las columnas 1-3-6. Unidad de medida minutos.

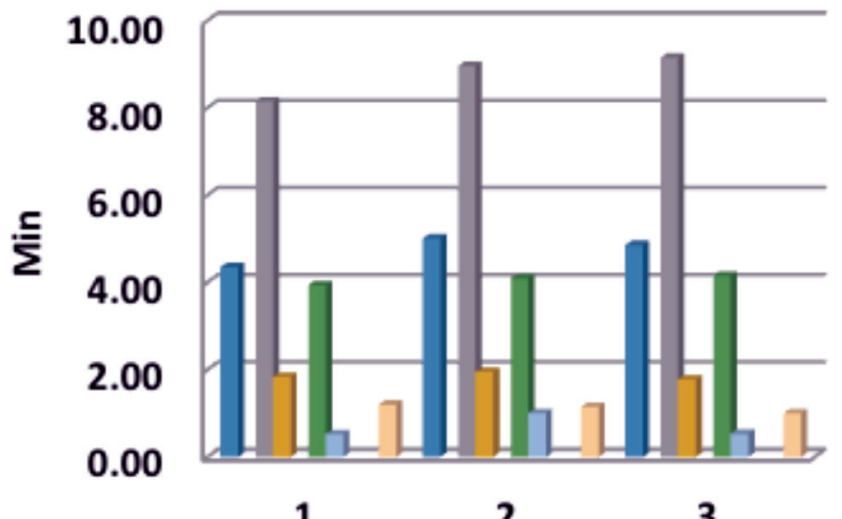

Fig. 12 Tendencia de las actividades que ocupan el mayor tiempo en el cambio de tintas 5-2016.

Fuente: elaboración propia

La Fig. 13 describe cada una de las tareas por medio del flujograma de procesos.

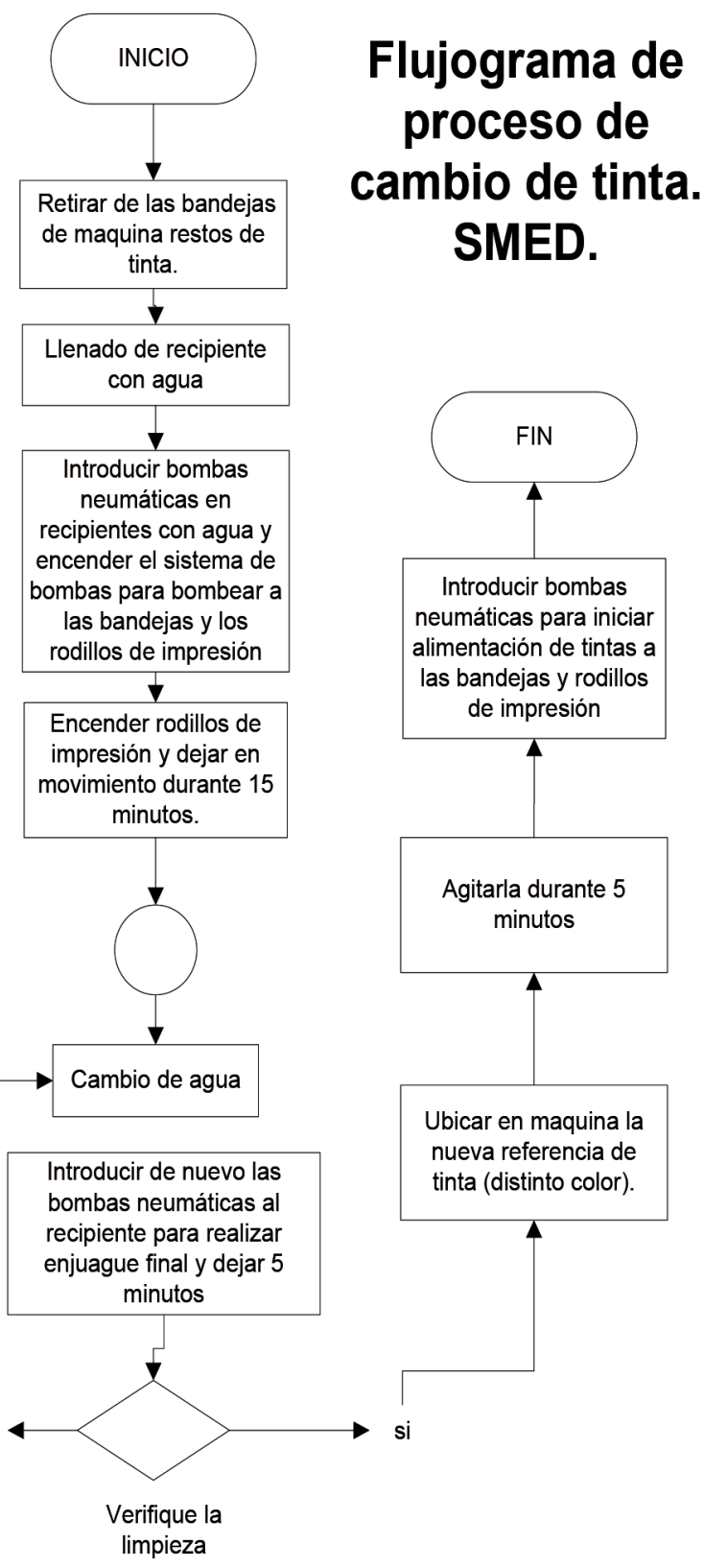

Fig. 13 Flujograma de procesos aplicando SMED. Fuente: elaboración propia

4) Tercera etapa: perfeccionar las tareas internas y externas.

\section{1) Análisis de datos}

En esta tabla se registran como evidencia del trabajo realizado los siguientes datos.

Datos iniciales de los cinco cambios tomados del 2015 como punto de partida donde esto consumía un promedio por cambio de $127.4 \mathrm{~min}$. 
Luego encontramos que con la implementación de la técnica Smed y la estandarización del proceso.

Finalmente se muestra el tiempo promedio del cambio de tintas para estas referencias de $62.97 \mathrm{~min}$.

La Fig. 14 Muestra el comparativo de los tiempos iniciales VS los datos finales obtenidos.

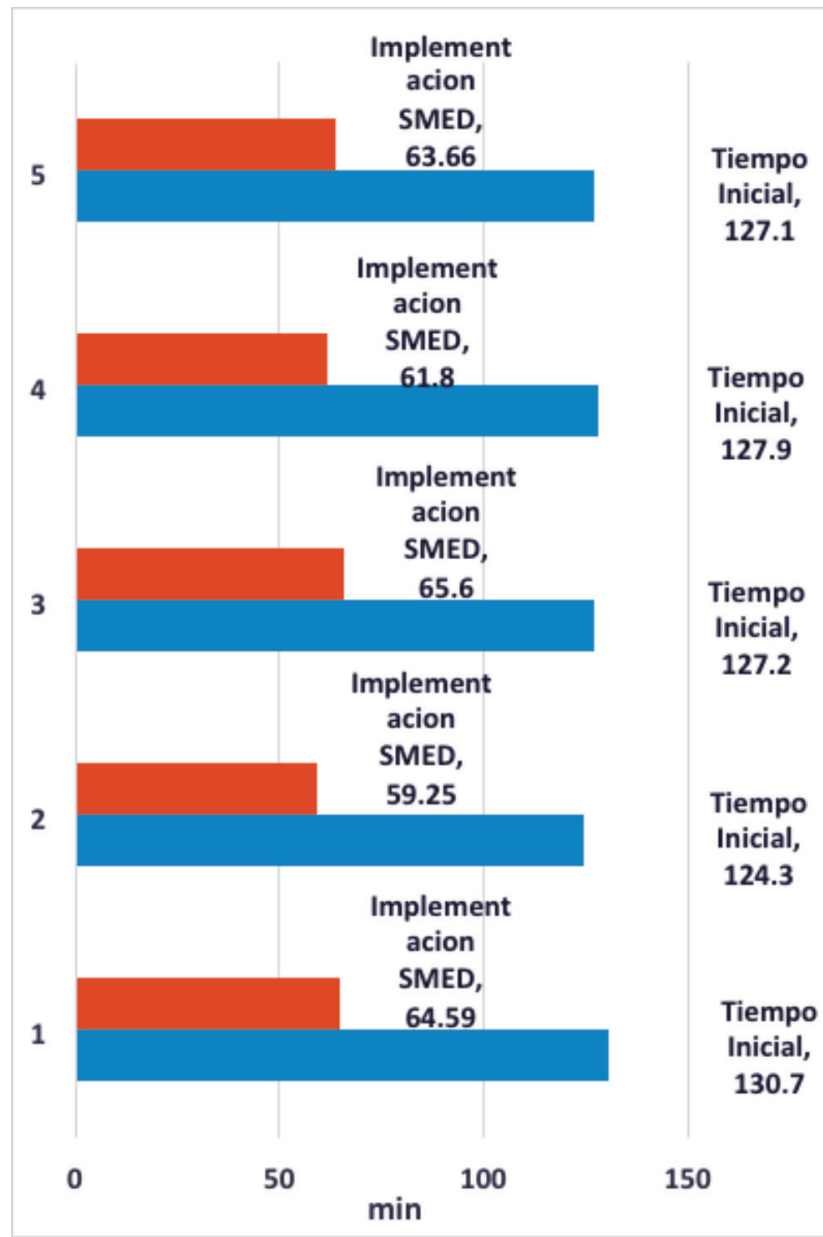

Fig. 14 Representación gráfica de la mejora obtenida, en minutos. Fuente: elaboración propia

\section{Conclusiones}

Al cambiar el método para el cambio de tintas de la referencia bolsa Kraff Colanta Entera 3C a bolsa Kraff Amtex Tannus 2C, se evidencia una mejora en disminución del tiempo de 23 minutos, un 18.3\% por cambio.
Después de separar y estandarizar las actividades internas de las actividades externas se encuentra una reducción del tiempo por cambio de tintas de la referencia bolsa Kraff Colanta Entera 3C a bolsa Kraff Amtex Tannus 2C, de 64 minutos, un $50.6 \%$ con relación al método inicial.

Además, se genera un impacto ambiental positivo ya que se reduce en un 30\% aproximadamente el consumo de agua por lo tanto reducción en la contaminación de esta.

\section{RECOMENDACIONES}

Se sugiere instalar un sistema de lavado automatizado para reducir aún más los tiempos de cambio de referencia de tintas.

Utilizar un producto de limpieza que acelere el desprendimiento de las tintas de los rodillos y elementos de máquina disminuyendo el tiempo de limpieza, consumo y contaminación de agua; con su s impactos ambientales.

Utilizar agitador eléctrico adecuado para homogenizar las tintas y mejorar la solubilidad de las tintas y su optimización en el proceso.

\section{REFERENCIAS}

Abraham, A, Ganapathi, K \& Motwani, J 2012, 'Setup time reduction through Smed technique in a stamping production line', SASTECH Journal, 11 (2), 47-52.

Almomani, M , Aladeemy, M, Abdelhadi, A \& Mumani, A 2013, A proposed approach for setup time reduction through integrating conventional Smed method with multiple criteria decision-making techniques', Computers \& Industrial Engineering, 66 (2), 461-469.

Azizi, A \& Manoharan, T 2015, 'Designing a future value stream mapping to reduce lead time using Smed -a case study', Procedia Manufacturing, 2, 153-158.

Kamath, N \& Rodrigues, L 2006, 'Simultaneous consideration of TQM and TPM influence on production performance: A case study on multicolor offset machine using SD Model', Perspectives in Science, 8, 16-18. 
- Fernando Ulpiano Pantoja Agreda, Jorge Humberto Castrillón

Aplicación de la técnica Smed en el procedimiento de cambio de tintas

de la referencia bolsa Kraff Colanta Entera 3C a bolsa Kraff Amtex Tannus 2C

Lipiak, J 2017, 'Methodology for assessing the factors affecting the quality and efficiency of flexographic printing process', Procedia Engeneering,182, 403-411.

Marsh, K \& Bugusu, B 2007, 'Food packaging - roles, materials, and environmental issues: scientific status summary', Journal of Food Science, 72 (3), 39-55.

Rentzhog, M \& Fogden, A 2006, 'Print quality and resistance for water-based flexography on polymer-coated boards: dependence on ink formulation and substrate pretreatment', Progress in Organic Coatings, 57 (3), 183-194.

Sendoya, F 2013, '¿ Qué es el control predictivo y hacia dónde se proyecta ?’, Revista Publicaciones e Investigación, Unad, p. 54-59.
Suárez, M \& Dávila, M 2008, 'Encontrando al Kaizen: un análisis teórico de la mejora continua," Pecunia Revista de la Facultad de Ciencias Económicas y Empresariales, (7), 285-311.

Sugimori, Y, Kusunoki, K \& Oho, F 1997, 'Toyota production system and Kanban system Materialization of just-in-time and respect-for-human system', International Journal of Production Research, 15 (6), 553-564.

Ulutas, B 2011, 'An application of Smed methodology', International Journal of Industrial and Manufacturing Engineering, 5 (7), 100-103. 


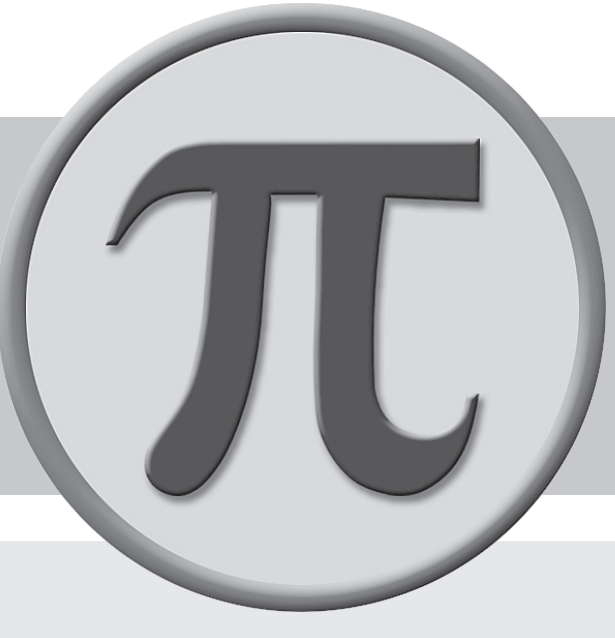


